\title{
Eating Disorder Examination Questionnaire (EDE-Q) and Clinical \\ Impairment Assessment (CIA) - Clinical Norms and Functional Impairment in Male and Female Adults with Eating Disorders
}

Camilla Lindvall Dahlgren ${ }^{\mathrm{a}^{*}}$, Kristin Stedal ${ }^{\mathrm{a}} \& \emptyset_{\mathrm{yvind}} \varnothing^{\mathrm{a}, \mathrm{b}}$

${ }^{\text {a }}$ Regional Department for Eating Disorders, Division of Mental Health and Addiction, Oslo University Hospital, Ullevål HF, Postboks 4950 Nydalen, 0424 Oslo, Norway

${ }^{\mathrm{b}}$ Institute of Clinical Medicine, University of Oslo, P.O. Box 1171 Blindern, 0318 Oslo, Norway

*Corresponding author: Camilla Lindvall Dahlgren. Regional Department for Eating Disorders, Division of Mental Health and Addiction, Oslo University Hospital, Ullevål HF, Postboks 4950 Nydalen, 0424 Oslo, Norway. Tel: (+47) 47291950, Email: camilla.lindvall@dahlgren.no 


\section{Abstract}

The aim of the current study was to collect clinical normative data for the Clinical Impairment Assessment questionnaire (CIA) and the Eating Disorder Examination Questionnaire (EDE-Q) from adult patients with eating disorders (EDs). This study also examined unique contributions of eating disorder (ED) symptoms on levels of ED-related impairment. A sample of 667 patients, 620 females and 47 males, was recruited from six specialist centres across Norway. The majority of the sample (40.3\%) was diagnosed with eating disorder not otherwise specified (EDNOS), $34.5 \%$ had bulimia nervosa $(\mathrm{BN})$ and $25.2 \%$ were diagnosed with anorexia nervosa $(\mathrm{AN})$. There were significant differences for global EDE-Q and CIA scores between females and males. In the female sample, significant differences were found on several EDE-Q subscales between the AN and BN group, and between the AN and EDNOS group. No significant differences were found between the diagnostic groups on the CIA. In the male sample, no significant differences were found between diagnostic groups on the EDE-Q or CIA. A multiple regression analysis revealed that $46.8 \%$ of the variance in impairment as measured by the CIA was accounted for by ED symptoms. Body mass index, eating concern, shape/weight concern and binge eating served as significant, unique predictors of impairment. The results from the present study contribute to the interpretation of EDE-Q and CIA scores in ED samples.

Keywords: Eating Disorders, Anorexia Nervosa, Bulimia Nervosa, Eating Disorder Examination Questionnaire (EDE-Q), Clinical Impairment Assessment (CIA), Norms 


\section{Introduction}

Structured clinical interviews are widely viewed as the most accurate method to determining eating disorder (ED) diagnoses and when assessing illness severity. They are designed to help identify core ED pathology, an essential first step in informing treatment. Although clinical interviews provide in-depth information about ED specific cognitions and behaviours, they have the disadvantage of being time consuming and costly to administer. The development and use of ED-specific self-report questionnaires has helped alleviate some of these drawbacks, with two of the most frequently-used questionnaires being the Eating Disorder Examination Questionnaire (EDE-Q) (1) and the Clinical Impairment Assessment (CIA) questionnaire (2). Whereas the EDE-Q was developed as a self-report version of the Eating Disorder Examination Interview (3) to measure key attitudes and behavioural features of EDs, the CIA was designed to assess functional impairment related to EDs. The CIA is intended to supplement the EDE-Q by providing information about impairment secondary to eating disorder symptoms.

There exist large sets of normative data collected from community and population-based samples for the EDE-Q (4-10) and to a lesser extent, the CIA $(2,11,12)$. However, little is known about the distribution of scores among individuals with EDs in clinical samples, and even less is known in terms of the diagnostic distribution of scores within and between the various ED diagnoses. Further, with a few exceptions (13-15), existing data from the EDE-Q and the CIA have been collected for female samples (16-21), leaving behavioural features, attitudes and ED-related impairment among males relatively unexplored. As clinical norms are important for treatment planning and the measurement of clinically significant change (22), and as no such norms exist 
for the Norwegian versions of the EDE-Q and CIA, the primary aim of our study was to establish clinical norms for the EDE-Q and the CIA using data collected from male and female patients presenting with a range of diagnoses based upon the DSM-IV (23).

Although the CIA was developed as a supplement to the EDE-Q, and should be used in tandem $(2,24)$ there are surprisingly few studies investigating the association between ED symptoms and functional impairment. To the authors' knowledge, merely one previously published study has investigated the impact of ED psychopathology (as assessed using the EDE-Q) on psychosocial impairment (as assessed using the CIA) (25). Due to the lack of research on this topic, we know little about how the observed impairment in EDs compares between different ED diagnoses, and whether certain ED symptoms have a greater impact on impairment compared to others.

Consequently, the second aim of our study was to investigate how individual ED symptoms and features contribute to functional impairment in patients diagnosed with different ED diagnoses.

\section{Methods}

\section{Participants and Procedure}

Participants were inpatients and outpatients recruited from six specialist eating disorder centres in Norway, who completed the EDE-Q and CIA questionnaire at the start of treatment. All patients met ICD-10 criteria for an eating disorder (F50.0-F50.9). The sample consisted of 667 patients (93\% females and 7\% males), with a mean age of $28.2(S D=8.6$, range 16-61) years. For this study, ICD-10 diagnoses were transformed to DSM-IV diagnoses, resulting in a 
diagnostic distribution of $25 \%$ patients with anorexia nervosa (AN), $35 \%$ with bulimia nervosa (BN) and 40\% with eating disorder not otherwise specified (EDNOS). The EDNOS category $(\mathrm{N}=269)$ is a summary of the following diagnostic categories: F50.1 (Atypical AN), $\mathrm{N}=117$, F50.3 (Atypical BN), N=64, F50.4 (Overeating associated with other psychological disturbances), N=2, F50.5 (Vomiting associated with other psychological disturbances), N=1, F50.8, N=21 (Other Specified ED), N=21 and F50.9 (Unspecified ED), N=64. Diagnostic and demographic information was collected from medical records. The Norwegian Regional Committee for Medical and Health Research Ethics approved the study.

\section{Measures}

The Eating Disorder Examination Questionnaire (EDE-Q, v. 16.0) (1) measures attitudinal features of eating disorders and core eating disorder behaviours (binge eating and inappropriate compensatory behaviours) over the past 28 days. It has four clinically-derived subscales each consisting of five to eight items: dietary restraint, eating concern, weight concern, and shape concern, which are used to calculate a global EDE-Q score. The 22-attitudinal items that comprise the global score are each rated using seven-point forced-choice format (0-6), with higher scores reflecting greater pathology. The Norwegian translation of the EDE-Q has demonstrated convergent validity with the EDE-interview (2) in a non-clinical sample of female university students, and similar to a recent, Finnish, validation (26), satisfactory internal consistency for both the global $(\alpha=.94)$, and subscales scores for restraint $(\alpha=.75)$, eating concern $(\alpha=.78)$, shape concern $(\alpha=.90)$ and weight concern $(\alpha=.81)$ (7). The internal 
consistency in this study was also satisfactory (global EDE-Q $\alpha=.94$, restraint $\alpha=.82$, eating concern $\alpha=.75$, shape concern $(\alpha=.88)$, and weight concern $(\alpha=.82)$.

The Clinical Impairment Assessment (CIA, version 3.0) questionnaire is a 16-item self-report measure of functional impairment secondary to eating disorder psychopathology (2). Items probe impairment in life domains typically affected by EDs such as mood, self-perception, cognitive functioning, interpersonal functioning and work performance over the past 4 weeks. Respondents rate items using a 4-point scale with responses ranging from "not at all" to 'a lot", providing both general and domain-specific indices of functional impairment. A global score and three subscale scores (personal impairment, cognitive impairment, and social impairment) can be calculated. Total scores range from 0 to 48 , with higher scores representing greater impairment. The Norwegian version has shown satisfactory psychometric properties and excellent internal consistency for the global score $(\alpha=.92)$, as well as for the three subscale scores personal, social and cognitive impairment $(\alpha=.93, \alpha=.84$, and $\alpha=.86)$, respectively (11) in a non-clinical sample of young adult females recruited at universities in Norway. In this study the internal consistency was also satisfactory (CIA global score $\alpha=.92$, personal impairment $\alpha=.83$, social impairment $\alpha=.85$, cognitive impairment $\alpha=.85$ ).

\section{Statistical Analyses}

All statistical analyses were performed using IBM SPSS Statistics 21. Student t-test and Pearson's chi-square test were used to explore differences between groups. Analysis of variance (ANOVA) was used to explore differences of EDE-Q and CIA scores between all diagnostic groups for each gender separately. The post hoc Scheffé method was used to investigate 
significant differences between specific diagnostic groups. Effect sizes were calculated using Cohen's $d$ (27) (small $d=0.2$, medium $d=0.5$ and large $d=0.8$ ) and partial eta-squared $\left(\eta_{p}^{2}\right)$

for analysis of variance ( small $\eta_{p}{ }^{2}=.01$, medium $\eta_{p}{ }^{2}=.06$, and large $\eta_{p}{ }^{2}=.14$ ). We used a hierarchical multiple regression analysis to explore the influence of unique individual ED symptoms on ED- related impairment. The CIA global score was used as the dependent variable, with BMI, the EDE-Q subscales eating concern and restraint, as well as the EDE-Q items on binge eating, self-induced vomiting, and laxative misuse, entered as independent variables. Because of a high inter-correlation (Pearson $r=.88$ ), the EDE-Q subscales weight concern and shape concern were averaged together to create an aggregate weight/shape concern variable. Gender and age were added to the model to control for the influence of these (potential) confounders. Statistical significance was set at the conventional level of $p<.05$ for all model parameters.

We used the thresholds reported in Welch et al. (28) for occurrence of disordered eating behaviour (see Table 3). Not all patients responded to the EDE-Q items on dietary restraint, excessive exercise, objective binge eating, self-induced vomiting and laxative misuse. Consequently, results were presented in valid percent (i.e. percentage that does not include missing cases).

\section{Results}

\section{Demographics and diagnostic distribution}

Table 1 presents the demographics of the female and male samples. No significant gender 
differences were found for age or BMI $(p>.05)$.

[Insert Table 1 here]

\section{$\underline{\text { EDE-Q and CIA scores }}$}

Means and standard deviations for the EDE-Q and the CIA scores for the female and male sample are presented in Table 2. Males scored significantly lower compared to females on the EDE-Q global score $(\mathrm{t}(51.28)=2.29, p=.026, d=.37)$ as well as on the weight concern $(\mathrm{t}(665)$ $=2.22, p=.027, d=.33)$, shape concern $(\mathrm{t}(665)=2.61, p=.009, d=.39)$, and eating concern $(\mathrm{t}(665)=2.25, p=.005, d=.40)$, EDE-Q subscales but not on restraint subscale $(\mathrm{t}(665)=1.51$, $p=.133, d=.21)$. Using Bonferroni correction of multiple comparisons for the four subscales $(p<.013)$ only shape and eating concern were statistically different between genders. Males also scored significantly lower on the CIA global score $(\mathrm{t}(49,88)=2,19, p=.033, d=0.37)$ and on the CIA subscales personal $(\mathrm{t}(49.52)=2.17, p=.035, d=.37$, and cognitive impairment $(\mathrm{t}(597)$ $=3.03, p=.005, d=.46)$, but not on the social impairment subscale $(\mathrm{t}(49.82)=0.86, p=.393$, $d=.15)$. Using Bonferroni correction of multiple comparisons for the three subscales $(p<.017)$ only cognitive impairment was statistically different between genders.

One-way analysis of variance (ANOVA) showed a significant difference in EDE-Q global scores between diagnostic groups among females $\left(\mathrm{F}(2,617)=8.37, p<.001, \eta_{p}{ }^{2}=.03\right)$ but no significant differences between diagnostic groups in males $\left(\mathrm{F}(2,44)=0.10, p=.91, \eta_{p}{ }^{2}=.004\right)$. The multivariate analysis of variance (MANOVA) indicated an overall effect for EDE subscales

for females, Wilks' Lambda $=.62, \mathrm{~F}(8,1230)=6.65, p<.001, \eta_{p}{ }^{2}=.04$. Using univariate 
analyses for the female sample, there were significant differences between diagnostic groups $(p<.001)$ for eating concern $\left(\mathrm{F}(2,617)=11.38, p<.001, \eta_{p}{ }^{2}=.04\right)$, shape concern $(\mathrm{F}(2,617)=$ $\left.6.58, p=.001, \eta_{p}{ }^{2}=.02\right)$ and weight concern $\left(\mathrm{F}(2,617)=12.90, p<.001, \eta_{p}{ }^{2}=.04\right)$ subscales. Post hoc tests showed significantly lower subscale scores ( 3 of 4 subscales) in the AN group compared to the $\mathrm{BN}$ group $(p<.05-p<.001)$, and lower scores in the AN group compared to the EDNOS group $(p<.01-p<.001)$ in the female sample. There were no statistical differences for CIA total score between diagnostic groups among females $\left(\mathrm{F}(2,550)=1.57, p=.21, \eta_{p}{ }^{2}=.006\right)$ and males $\left(\mathrm{F}(2,43)=1.00, p=.38, \eta_{p}^{2}=.04\right)$.

\section{[Insert Table 2 here]}

\section{Occurrence of eating disorder behaviors}

Overall, participants reported a mean $(S D)$ of 13.8 (26.4) binge eating episodes, $19.0(34.4)$ vomiting episodes, $2.0(6.2)$ episodes of laxative misuse and 12.5 (15.4) episodes of excessive exercise over the past 28 days. Table 3 presents the proportion of females and males respectively, engaging in the following eating disordered behaviours: dietary restraint, episodes of excessive exercise, objective binge eating, self-induced vomiting and laxative misuse. Using Chi-square test, there were no statistical differences between genders in the proportion engaging in different eating disorder behaviors.

[Insert Table 3 here] 


\section{Contributions of ED symptoms to ED- related impairment}

The results from the regression analyses revealed that the first model (age and gender) accounted for a small $(1.8 \%)$ contribution to the variance in the CIA total score and only gender had a significant contribution $(p<.01)$. After the entry of the seven independent variables (BMI, EDEQ subscales eating concern, restraint and shape/weight concern, binge eating, self-induced vomiting, and laxative misuse), the total variance explained by the second model as a whole was $46.8 \%(p<.001)$, with the seven variables explaining an additional $44.9 \%$ of the variance in EDassociated impairment beyond age and gender. In the third model only significant variables in model two were included (BMI, binge eating, EDE-Q subscales eating concern, restraint and shape/weight concern) and the results showed that restraint subscale did not have a significant contribution to global CIA. The final fourth model with significant variables in model three (BMI, binge eating, EDE-Q subscales eating concern and shape/weight concern) made unique, significant contributions to the level of impairment assessed by the CIA total score and explained $46.5 \%$ of the variance. As shown in table 4, eating concern had the biggest contribution to CIA total score (highest standardized beta value) followed by shape/weight concern, BMI and binge eating episodes in the final model 4.

[Insert Table 4 here]

\section{Discussion}

The primary aim of the current study was to establish clinical norms for the EDE-Q and the CIA for male and female in- and outpatients diagnosed with an ED. Male patients scored significantly lower on both the EDE-Q and CIA than females. Female AN patients scored significantly lower than BN and EDNOS female patients on EDE-Q, but no difference in CIA scores were found 
between diagnostic groups. We also wanted to investigate how individual ED symptoms and features contributed to functional impairment, and our findings showed that approximately $50 \%$ of ED-related impairment could be explained by ED symptoms assessed using the CIA.

In Norway, community norms have been established for both the EDE-Q (31) and the CIA (11) in healthy female adults, but to date, there are no studies investigating norms in clinical ED samples. Although both Jenkins et al. (30) and Martin et al. (18) reported recruiting patients of both genders, our study is the first to present clinical CIA scores for both males and females. The results from the present study echo findings from a large study with 2383 participants from Sweden (28) in terms of diagnostic distribution, clinical EDE-Q norms, diagnostic differences in EDE-Q scores, and in the occurrence of binge eating episodes. In the Welch et al. study (28), males also scored significantly lower on EDE-Q than females. Similar to Welch and colleagues, results from our female sample revealed significant differences between the AN and BN diagnostic groups on all EDE-Q subscales scores except restraint, with the BN group scoring significantly higher on all subscales. Further, we also replicated Welch et al.'s results in failing to find a significant difference between the AN and EDNOS groups on the EDE-Q restraint subscale, and in reproducing their results reporting significant differences on all remaining EDEQ subscales except eating concern. In addition, the occurrence of vomiting and laxative misuse among females was less frequent in their study (55\% versus $62 \%$ and $12 \%$ versus $21 \%$, respectively). This was true for all diagnostic groups, and was also applicable to the male sample. 
In our female clinical sample, we found somewhat higher levels of ED impairment compared to previous reports $(2,7,28,31)$. Yet we reported a lower global CIA score compared to a UK clinical sample (30). These differences may be due to sample differences in treatment level, age, gender or diagnostic categories. Similar to Welch et al. (28), we found significantly lower scores on the CIA for males than for females. However, contrary to the findings by Welch and colleagues, who found lower CIA scores for individuals with EDNOS and BED, we failed to find significant differences between diagnostic categories. In our study, patients with BED were included in the EDNOS group since BED is not a formal diagnosis in the ICD-10. Although similar in diagnostic distribution, it should be noted that the Welch et al. sample was considerably larger than ours $(\mathrm{N}=2383$ vs. 667$)$, with a considerably lower proportion of males (2.4\% vs. $7.0 \%$ ) (19). Any similarities or differences between these two studies should therefore be interpreted carefully, potentially influenced by sample sizes and gender discrepancies.

When investigating the unique contributions of individual ED symptoms to impairment, our results revealed that nearly half (47\%) of the impairment experienced by our patients was directly attributable to ED psychopathology. Specifically, eating concern, weight/shape concern, BMI and binge eating made significant unique contributions to the regression model. These numbers correspond well to those reported in Hovrud and De Young (25), who found that ED psychopathology accounted for $46 \%$ of the variance in clinical impairment. They reported that following the inclusion of depression in the model, the total explained variance increased from $46 \%$ to $58.6 \%$, with binge eating, weight/shape concern and depression serving as unique predictors of impairment (18). Unfortunately, we were unable to assess depression in our study, and thus, were unable to explore its predictive value on ED-related impairment. Similar to 
Hovruds and De Young (25), binge eating and weight/shape concern were significant predictors of impairment in our model. In contrast, however, we found that eating concern and BMI accounted for unique variance in CIA scores. These differences may be due to the impact of depression in the Hovrud and De Young's regression analyses, and this warrants further investigation.

Although community norms have been established for both the EDE-Q (31) and the CIA (11) in Norway, this is the first study to report normative data in a clinical ED sample, and to further investigate the association between these two well-established clinical instruments. The present study included a large representative clinical sample, consisting of data collected from six treatment centres, including both female and male in- and outpatients. The study is further strengthened by the sample size, which is considerably larger than the majority of previously published studies on clinical CIA and EDE-Q norms. However, there are some limitations to consider. Diagnoses were clinically derived according to ICD-10 criteria and we lacked diagnostic data needed to establish AN subtypes. Also, the EDNOS group in the current study consists of a combination of residual ED diagnostic categories. Any inferences about, and comparisons to this group should therefore be made with caution. Results from the male sample should also be interpreted cautiously as reliable between subject differences are practically nondetectable in a sample size this small. We did not have data on comorbidity, and thus, we were unable to investigate the potential effects of comorbidity such as anxiety or depression on ED symptomatology and clinical impairment. 
In summary, our study contributes to the existing literature by reporting clinical EDE-Q and CIA normative data, and adds to the scant literature on males with EDs. Using the same methodology, we managed to replicate findings from the study by Hovrud and De Young (25), achieving nearly identical findings for the contribution of ED symptoms on impairment. Future research should aim to include more males with EDs, in addition to investigating other potential predictors such as depression and anxiety, which may contribute to everyday impairment in patients with ED. Improved knowledge of the factors associated with impairment will not only contribute to the interpretation of EDE-Q and CIA scores, but will also help inform treatment planning for individuals diagnosed with eating disorders.

\section{Acknowledgements}

The authors acknowledge the six participating eating disorder centres and their patients who helped fill out the self-report questionnaires. The authors would also like to thank Dr. Deborah L. Reas for proofreading the manuscript prior to resubmission.

\section{Conflicts of interest}

The authors declare that there are no conflicts of interest. 


\section{References}

1. Fairburn CG, Beglin SJ. Assessment of eating disorders: interview or self-report questionnaire? The International journal of eating disorders. 1994;16(4):363-70.

2. Bohn K, Doll HA, Cooper Z, O'Connor M, Palmer R, Fairburn C. The measurement of impairment due to eating disorder psychopathology. Behavior Research and Therapy. 2008;46(10):1105-10.

3. Fairburn CG, Cooper Z, O'Connor M. Eating Disorder Examination (16.0D). In: Fairburn CG, editor. Cognitive Behavior Therapy and Eating Disorders. New York: Guilford Press; 2008. 4. Nakai Y, Nin K, Fukushima M, Nakamura K, Noma S, Teramukai S, et al. Eating disorder examination questionnaire (EDE-Q): norms for undergraduate Japanese women. Eur Eat Disord Rev. 2014;22(6):439-42.

5. Machado PP, Martins C, Vaz AR, Conceicao E, Bastos AP, Goncalves S. Eating disorder examination questionnaire: psychometric properties and norms for the Portuguese population. Eur Eat Disord Rev. 2014;22(6):448-53.

6. Quick VM, Byrd-Bredbenner C. Eating Disorders Examination Questionnaire (EDE-Q): norms for US college students. Eat Weight Disord. 2013;18(1):29-35.

7. $\mathrm{R} \varnothing \varnothing$, Reas D, Lask B. Norms for the Eating Disorder Examination Questionnaire among female university students in Norway. Nordic Journal of Psychiatry. 2010;64(6):428-32.

8. Hilbert A, de Zwaan M, Braehler E. How frequent are eating disturbances in the population? Norms of the eating disorder examination-questionnaire. PLoS One. 2012;7(1):e29125. 
9. Pelaez-Fernandez MA, Labrador FJ, Raich RM. Norms for the Spanish version of the Eating Disorders Examination Questionnaire (S-EDE-Q). Psicothema. 2013;25(1):107-14.

10. Kelly NR, Cotter EW, Mazzeo SE. Eating Disorder Examination Questionnaire (EDEQ): norms for Black women. Eat Behav. 2012;13(4):429-32.

11. Reas D, Ro O, Kapstad H, Lask B. Psychometric properties of the clinical impairment assessment: norms for young adult women. Int J Eat Disord. 2010;43(1):72-6.

12. Vannucci A, Kass AE, Sinton MM, Aspen V, Weisman H, Bailey JO, et al. An examination of the Clinical Impairment Assessment among women at high risk for eating disorder onset. Behav Res Ther. 2012;50(6):407-14.

13. Mond J, Hall A, Bentley C, Harrison C, Gratwick-Sarll K, Lewis V. Eating-disordered behavior in adolescent boys: eating disorder examination questionnaire norms. Int J Eat Disord. 2014;47(4):335-41.

14. Reas D, Overas M, Ro O. Norms for the Eating Disorder Examination Questionnaire (EDE-Q) among high school and university men. Eat ing Disorders. 2012;20(5):437-43.

15. Lavender JM, De Young KP, Anderson DA. Eating Disorder Examination Questionnaire (EDE-Q): Norms for undergraduate men. Eating Behaviors. 2010;11(2):119-21.

16. Calugi S, Milanese C, Sartirana M, El Ghoch M, Sartori F, Geccherle E, et al. The Eating Disorder Examination Questionnaire: reliability and validity of the Italian version. Eat Weight Disord. 2016.

17. Becker AE, Thomas JJ, Bainivualiku A, Richards L, Navara K, Roberts AL, et al. Adaptation and evaluation of the Clinical Impairment Assessment to assess disordered eating related distress in an adolescent female ethnic Fijian population. Int J Eat Disord. 2010;43(2):179-86. 
18. Martin J, Padierna A, Unzurrunzaga A, Gonzalez N, Berjano B, Quintana J. Adaptation and validation of the Spanish version of the Clinical Impairment Assessment Questionnaire. Appetite. 2015;91:20-7.

19. Luce KH, Crowther JH, Pole M. Eating Disorder Examination Questionnaire (EDE-Q): norms for undergraduate women. Int J Eat Disord. 2008;41(3):273-6.

20. DeJong H, Oldershaw A, Sternheim L, Samarawickrema N, Kenyon MD, Broadbent H, et al. Quality of life in anorexia nervosa, bulimia nervosa and eating disorder not-otherwisespecified. Journal of eating disorders. 2013;1:43.

21. Reas DL, Ro O, Kapstad H, Lask B. Psychometric properties of the clinical impairment assessment: norms for young adult women. Int J Eat Disord. 2010;43(1):72-6.

22. Mitchison D, Morin A, Mond J, Slewa-Younan S, Hay P. The bidirectional relationship between quality of life and eating disorder symptoms: a 9-year community-based study of Australian women. PLoS One. 2015;10(3):e0120591.

23. American Psychiatric Association. Diagnostic and Statistical Manual of Mental Disorders DSM-IV (4th ed.). Washington, DC: American Psychiatric Association; 1994.

24. Center for Reserach on Eating Disorders (CREDO). The Clinical Impairment Assessment (CIA) http://credo-oxford.com/7.2.html: http://www.credo-oxford.com;/ 2016

25. Hovrud L, De Young KP. Unique contributions of individual eating disorder symptoms to eating disorder-related impairment. Eating Behaviors. 2015;18:103-6.

26. Isomaa R, Lukkarila IL, Ollila T, Nenonen H, Charpentier P, Sinikallio S et al. Development and preliminary validation of a Finnish version of the Eating Disorder Examination Questionnaire (EDE-Q). Nordic Journal of Psychiatry. 2016;(70)7:542-546.

27. Cohen, J. Statistical power analysis for the behavioral sciences (2nd ed.). Hillsdale, 
NJ: Lawrence Earlbaum Associates; 1988.

28. Welch E, Birgegård A, Parling T, Ghaderi A. Eating disorder examination questionnaire and clinical impairment assessment: General population and clinical norms for young adult women in Sweden. Behaviour Research and Therapy. 2011;49:85-91.

29. Rø Ø, Reas D, Stedal K. Eating Disorder Examination Questionnaire (EDE-Q) in Norwegian Adults: Discrimination between Female Controls and Eating Disorder Patients. European Eating Disorders Review. 2015;23(5):408-12.

30. Jenkins P. Psychometric validation of the Clinical Impairment Assessment in a UK eating disorder service. Eating Behaviors. 2013;14(2):241-3.

31. Ekeroth K, Birgegard A. Evaluating reliable and clinically significant change in eating disorders: comparisons to changes in DSM-IV diagnoses. Psychiatry research. 2014;216(2):248- 